\title{
Supporting information: \\ Squeezed light from entangled nonidentical emitters via nanophotonic environments
}

\author{
Harald R. Haakh and Diego Martín-Cano \\ Max Planck Institute for the Science of Light, Günther-Scharowski-Straße 1/24, D-91058 Erlangen, Germany.
}

(Dated: November 23, 2015)

\section{MASTER EQUATION IN MACROSCOPIC ELECTRODYNAMICS}

We use the approach of macroscopic quantum electrodynamics [1] to describe the behavior of $N$ two-level quantum emitters in an arbitrary environment. Each emitter is described by its transition dipole element $\boldsymbol{d}_{n}$, emitter operator $\hat{\sigma}_{n}$, and transition frequency $\omega_{n}$. Within the dipole, rotating wave, and Markov approximation the full positive frequency field operator is given by

$$
\begin{aligned}
\hat{\boldsymbol{E}}_{\text {tot }}^{+}(\mathbf{r}, t) & =\hat{\boldsymbol{E}}_{\text {free }}^{+}(\mathbf{r}, t)+\sum_{n=1}^{N} \hat{\boldsymbol{E}}_{n}^{+}(\mathbf{r}, t) \\
\text { with } \hat{\boldsymbol{E}}_{n}^{+}(\mathbf{r}, t) & =\boldsymbol{g}_{n}(\boldsymbol{r}) \hat{\sigma}_{n}(t) .
\end{aligned}
$$

Here, $\boldsymbol{g}_{n}(\boldsymbol{r}) \approx \frac{\omega_{0}^{2}}{c^{2} \varepsilon_{0}} \boldsymbol{G}\left(\boldsymbol{r}, \boldsymbol{r}_{n}, \omega_{0}\right) \cdot \boldsymbol{d}_{n}$ encodes the photon propagation in an arbitrary broadband environment via the classical dyadic Green's tensor, where $\boldsymbol{r}$ denotes the detector position. As long as dispersion in a nanostructure is weak, we can evaluate the Green's tensor at the center frequency $\omega_{0}=\sum_{n} \omega_{n} / N$. Since we consider far-field observation, we neglect a small position-dependent quantum correction connected with dispersion potentials, which would require some care in the study of near-fields [2]. For a gold nanosphere, we evaluate the Green's function semi-analytically [3, 4], using a Drude-Lorentz interpolation of tabulated optical data for gold [5].

Under these conditions, the evolution equation is expressed in the interaction picture with respect to the free quantum fields and in the rotating frame of a classical driving field $E_{L}(\boldsymbol{r}) e^{-\mathrm{i} \omega_{L} t}+h . c$. that translates to single-emitter Rabi frequencies $\Omega_{n}=E_{L}\left(\boldsymbol{r}_{n}\right) \cdot \boldsymbol{d}_{n} / \hbar$. The Hamiltonian reads

$$
H=\hbar \sum_{n}\left[\left(\omega_{n}-\omega_{L}\right) \sigma_{z, n}-\left(\frac{\Omega_{n}}{2} \sigma_{n}^{+}+h . c .\right)\right]
$$

with $\sigma_{z, n}$ the population inversion of particle $n$. The quantum optical master equation in the Lindblad form is given by (see, e.g., Refs. [6, 7])

$$
\begin{gathered}
\dot{\rho}=-\frac{\mathrm{i}}{\hbar}\left[\left(H-\sum_{m \neq n} \Omega_{m n} \sigma_{m}^{\dagger} \sigma_{n}\right), \rho\right]+\mathcal{L}[\rho], \\
\mathcal{L}[\rho]=\sum_{m, n}-\frac{\gamma_{m n}}{2}\left(\sigma_{m}^{\dagger} \sigma_{n} \rho+\rho \sigma_{m}^{\dagger} \sigma_{n}-2 \sigma_{m} \rho \sigma_{n}^{\dagger}\right) .
\end{gathered}
$$

Photon-mediated interactions result in modified dipole-dipole potentials $\Omega_{m n}$ while the dissipative dynamics, i.e. the individual and cooperative decay of dipole excitations, are described by the Liouvillian $\mathcal{L}$. Here, the rates are the real and imaginary parts of

$$
\Omega_{m n}+\mathrm{i} \gamma_{m n} / 2=\boldsymbol{d}_{m} \cdot \boldsymbol{g}_{n}\left(\mathbf{r}_{m}\right)
$$

even for far-detuned emitters as long as $\omega_{1}, \omega_{2} \gg \delta$ [6, 7]. Due to the local field enhancement, the single-body decay rates in the nanostructure $\gamma_{m} \equiv \gamma_{m m}$ generally differ from the free-space value $\gamma_{0}$. For this work, the master equation (4) is solved numerically for the steady state solution in the case of $N=2$. Additional pure dephasing at a rate $\gamma_{m}^{*}$ can be included phenomenologically [8] by adding the term

$$
\mathcal{L}_{\mathrm{d}}[\rho]=\sum_{m}-\gamma_{m}^{*}\left(\sigma_{z, m} \sigma_{z, m} \rho+\rho \sigma_{z, m} \sigma_{z, m}-2 \sigma_{z, m} \rho \sigma_{z, m}\right)
$$

To obtain the solution and the relevant correlation functions, we employ the single-body basis $|1\rangle=\left|g_{1} g_{2}\right\rangle,|2\rangle=$ $\left|g_{1} e_{2}\right\rangle,|3\rangle=\left|e_{1} g_{2}\right\rangle,|4\rangle=\left|e_{1} e_{2}\right\rangle$, where $e_{i}\left(g_{i}\right)$ denotes the $i$-th emitter in the excited (ground) state. 
To describe the physics involving the weakly driven two-photon transition, it is insightful to use the collective coupled eigenstates in the absence of driving. For two coupled emitters detuned by $\delta=\omega_{2}-\omega_{1}$ and in the absence of driving, the coherent terms in the master equation (4) are diagonalized by the states

$$
\begin{array}{rlrl}
|G\rangle & =|1\rangle, & |E\rangle & =|4\rangle, \\
|S\rangle & =a|3\rangle+b|2\rangle, & |A\rangle & =b|2\rangle-a|3\rangle, \\
a & =\frac{d}{\sqrt{d^{2}+\Omega_{12}^{2}}}, & b & =\frac{\Omega_{12}}{\sqrt{d^{2}+\Omega_{12}^{2}}} \\
d & =2 \delta+\sqrt{4 \delta^{2}+\Omega_{12}^{2}} . &
\end{array}
$$

The corresponding eigenfrequencies $\omega_{E}=-\omega_{G}=\left(\omega_{1}+\omega_{2}\right) / 2, \omega_{S}=-\omega_{A}=\sqrt{\Omega_{12}^{2}+4 \delta^{2}}$ provide the level scheme shown in Fig. 1a in the main text and describe well the spectrum encountered at sufficiently weak drivings. In the case of identical particles $\delta=0, \gamma_{1}=\gamma_{2}$, the coupled basis recovers the bright and dark maximally entangled Dicke states $(|S\rangle,|A\rangle \rightarrow|+\rangle,|-\rangle)$ with decay rates $\gamma_{ \pm}=\gamma \pm\left|\gamma_{12}\right|$, respectively. These states govern the dynamics at weak driving [6, 9], whereas in the detuned case $\delta \neq 0,|G\rangle$ and $|E\rangle$ are mainly responsible for the response at the two-photon resonance as discussed in the main text.

\section{NANOSTRUCTURE MEDIATED DIPOLE-DIPOLE COUPLING: PARAMETER DEPENDENCE AND EFFECT OF QUENCHING}

The enhancement of the dipole-dipole coupling $\Omega_{12}$ due to the nanosphere depends on the position of the emitters with respect to the particle, the radius, and the center wavelength. In order to illustrate the robustness of this enhancement against variations of these parameters, we show in Fig $\mathrm{S} 1 \Omega_{12}$ as a function of the emitters-nanosphere distance $a$ and we consider three representative wavelengths and two nanoparticle radii $[R=20 \mathrm{~nm}$, panels (a)-(c), and $R=40 \mathrm{~nm}$, panels (d)-(f)]. All panels show that, at sufficiently small distances, the dipole-dipole coupling (black curve) gets significantly enhanced in comparison with the free-space coupling (black dashed-dotted line) and can reach a factor of $\Omega_{12} / \Omega_{12,0} \approx 3$ for the case considered in the main text [see Fig[S1 (f) at $a=25$ nm]. This enhancement gets stronger at decreasing separations, e.g. at $a=10 \mathrm{~nm}$, at the expense of increasing the non-radiative decay rate of the emitters, i.e., quenching. The onset of ohmic fluorescence quenching can be seen as an abrupt increase in the decay rate of the emitter $\gamma$ (blue dotted curve) at smaller $a$, which eventually surpasses $\Omega_{12}$ and gradually reduces the excitation of the two-photon coherence discussed in this work. Nevertheless we can observe that for both particle sizes, longer wavelengths facilitate enhanced dipole-dipole coupling $\Omega_{12}>\gamma$ via the nanosphere over spatial ranges of several tens of nanometers. This is in contrast to frequencies near the dipolar surface plasmon resonance (at $\lambda \approx 520$ $\mathrm{nm}$ ), where the increased ohmic absorption dominates over the coherent dipole-dipole coupling [panels (a) and (d)]. As the plasmon resonance wavelength increases with the particle size, similar effects can be extended to larger particles at increasing center wavelengths. The coupling constants also vary slowly on wavelength scales of tens of nanometers (not shown) due to the broadband character of the plasmon resonance resulting in an overall robustness of the scheme and the effects described in the main text.

\section{INCOHERENT FLUORESCENCE}

One relevant experimental observable used to identify the two-photon resonance is the incoherent fluorescence as a function of the driving frequency [10, which can be measured outside the spectral range of the exciting laser. By assessing where the fluorescence level is still low, one can identify the parameters for which coherent effects are expected to be significant. The fluorescence signal is proportional to $I=\sum_{i}\left\langle\hat{\boldsymbol{E}}_{i}^{-} \cdot \hat{\boldsymbol{E}}_{i}^{+}\right\rangle=\sum_{i}\left|g_{i}(\boldsymbol{r})\right|^{2}\left\langle\sigma_{i}^{\dagger} \sigma_{i}\right\rangle$. In the main text we set $\left|g_{1}(\boldsymbol{r})\right|=\left|g_{2}(\boldsymbol{r})\right|$, which is a reasonable assumption when the emitters are placed at equivalent positions with respect to the nanostructure. This can be inferred from Eqs. (1), (6), where for similar emitter positions the one-point Green's tensor provides $\boldsymbol{G}\left(\boldsymbol{r}_{1}, \boldsymbol{r}_{1}, \omega_{0}\right) \approx \boldsymbol{G}\left(\boldsymbol{r}_{2}, \boldsymbol{r}_{2}, \omega_{0}\right)$ and at equivalent far-field detection distances $\boldsymbol{G}\left(\boldsymbol{r}, \boldsymbol{r}_{1}, \omega_{0}\right) \approx \boldsymbol{G}\left(\boldsymbol{r}, \boldsymbol{r}_{2}, \omega_{0}\right)$. Nevertheless, we have numerically checked that values differing by up to an order of magnitude between $\left|g_{1}(\boldsymbol{r})\right|^{2}$ and $\left|g_{2}(\boldsymbol{r})\right|^{2}$ and between $\gamma_{1}$ and $\gamma_{2}$, the impact of the two-photon transition remains qualitatively similar to the case shown in Fig. 1 in the main text. Mainly, this introduces an asymmetry in the fluorescence and a gives a stronger weight in intensity to the emitter with the larger scattering amplitude $\left|g_{i}\right|$. On the other hand, the states $|G\rangle$ and $|E\rangle$ are still connected by a collective transition at $\Delta=\omega_{L}-\left(\omega_{1}+\omega_{2}\right) / 2=0$. This can be seen from the perturbative formula for the population of the excited state deduced from Ref. 11 

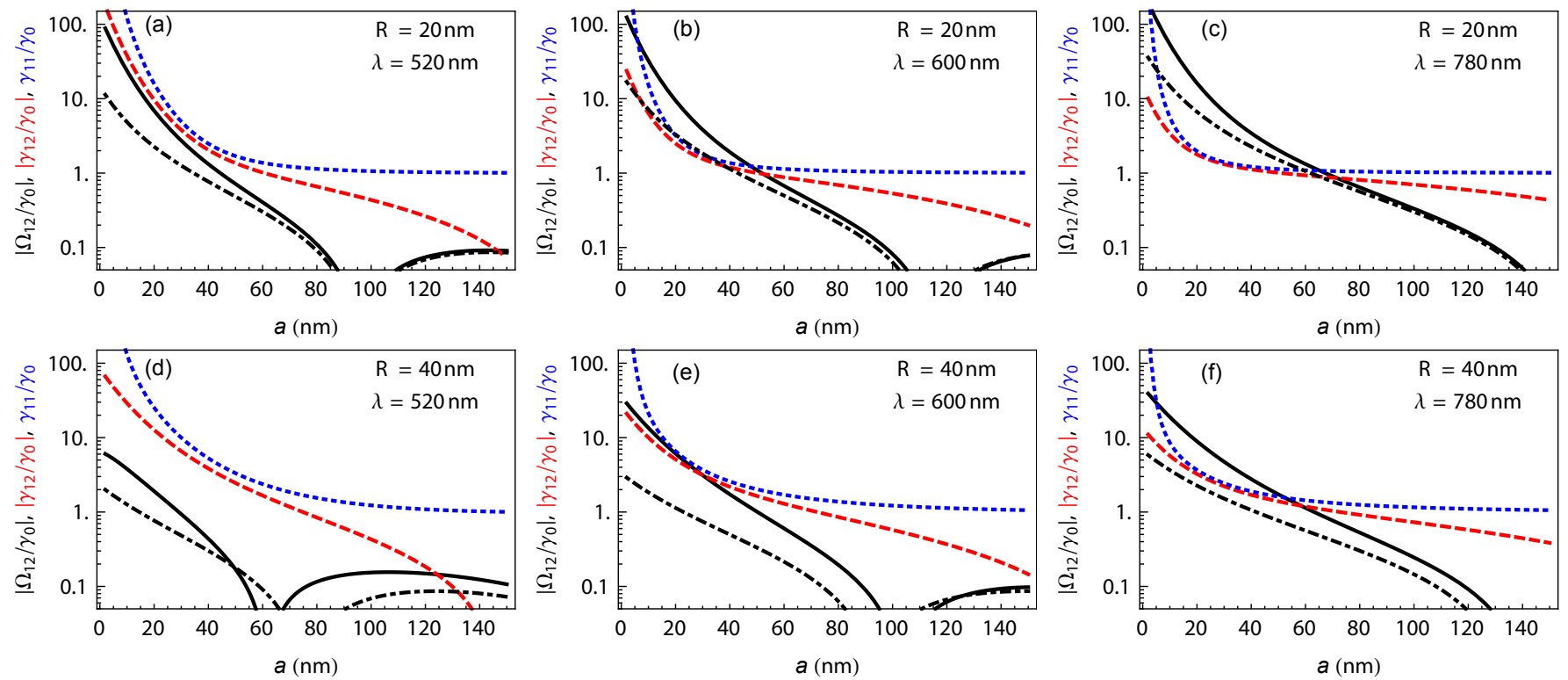

FIG. S1: Dipole-dipole coupling $\Omega_{12}$ (black line), incoherent coupling rate $\gamma_{12}$ (red dashed line) and emitter linewidth $\gamma$ (blue dotted line) versus the emitters distance $a$ at symmetric positions (see Fig. 1a in the main text). Panels (a)-(c) consider a gold nanosphere of $20 \mathrm{~nm}$ radius and center wavelengths $\lambda=2 \pi c / \omega_{0}$ of $\lambda=520 \mathrm{~nm}, 600 \mathrm{~nm}$ and $780 \mathrm{~nm}$, respectively. Panels (d)-(f) give the analogous representation for $R=40 \mathrm{~nm}$ considered in the main text. We consider radially oriented dipoles and the coupling constants are normalized to the decay rate in free space $\gamma_{0}$. The free-space contribution of the dipole-dipole coupling $\Omega_{12}$ is shown for comparison in every panel (black dashed-dotted line).

$$
\rho_{44}=\frac{1}{\left(\gamma_{1}+\gamma_{2}\right)^{2}+(2 \Delta)^{2}} \times\left|\frac{2 \Omega_{1} \Omega_{2}\left(\omega_{1}+\omega_{2}-2 \omega_{L}\right)}{\left(\omega_{1}-\omega_{L}\right)\left(\omega_{2}-\omega_{L}\right)}-\frac{\Omega_{12}\left[\Omega_{1}^{2}+\Omega_{2}^{2}\right]}{\left(\omega_{1}-\omega_{L}\right)\left(\omega_{2}-\omega_{L}\right)}\right|^{2}
$$

which can still provide comparable values for $\gamma_{1} \neq \gamma_{2}$ or asymmetric driving conditions $\Omega_{1} \neq \Omega_{2}$. In the latter case, the two-photon peak is shifted with respect to the symmetric scenario considered in the main text but without altering significantly the maximum values in either squeezing or entanglement.

\section{CONCURRENCE NEAR THE TWO-PHOTON RESONANCE}

The amount of entanglement generated in the coupled system may be quantified for a general quantum state using Wootters's concurrence [12] $C=\max \left[\left\{0, \lambda_{1}-\lambda_{2}-\lambda_{3}-\lambda_{4}\right\}\right]$,where the $\lambda_{n}^{2}$ are the eigenvalues of the operator $\rho\left(\sigma_{y, 1} \otimes \sigma_{y, 2}\right) \rho^{\star}\left(\sigma_{y, 1} \otimes \sigma_{y, 2}\right)$ in descending order. The ${ }^{\star}$ denotes the complex conjugate. To gain a better understanding of the entanglement induced by the two-photon resonance, we consider the limit of a weak driving field. In this case, the solution will be close to that of an undriven system, for which exact analytical solutions are available. Here, the density matrix keeps a cross-shaped symmetry [13] and the concurrence is expressed as

$$
C \approx \max \left\{0, C_{1}, C_{2}\right\} \text { with } \quad C_{1}=2\left|\rho_{41}\right|-2 \sqrt{\rho_{22} \rho_{33}}, \quad C_{2}=2\left|\rho_{23}\right|-2 \sqrt{\rho_{11} \rho_{44}} .
$$

We have checked for our scheme that $C_{1}$ applies near the two-photon resonance at weak driving, where the singlephoton populations are negligible. This shows that entanglement is due to the driving of the two-photon coherence $\rho_{14}$ connecting the double ground and double excited states. When $\left|\gamma_{12}\right|>0$, the asymmetry between the dark and bright states is visible in a dispersive contribution to the concurrence at larger positive detunings, for which then $C_{2}$ dominates. 


\section{ELECTRIC FIELD QUADRATURE FLUCTUATIONS}

We identify sub-shot-noise fluctuations in an electric field quadrature component $\hat{\boldsymbol{E}}_{\theta}=e^{\mathrm{i} \theta} \hat{\boldsymbol{E}}^{+}+e^{-\mathrm{i} \theta} \hat{\boldsymbol{E}}^{-}$by negative values of its normally ordered variance 14

$$
\left\langle:\left[\Delta \hat{\boldsymbol{E}}_{\theta}(\boldsymbol{r}, t)\right]^{2}:\right\rangle=2 \operatorname{Re}\left[e^{\mathrm{i} 2 \theta}\left\langle\left[\hat{\boldsymbol{E}}^{+}(\mathbf{r}, t)\right]^{2}\right\rangle-e^{\mathrm{i} 2 \theta}\left\langle\hat{\boldsymbol{E}}^{+}(\mathbf{r}, t)\right\rangle^{2}\right]+2\left\langle\hat{\boldsymbol{E}}^{-}(\mathbf{r}, t) \hat{\boldsymbol{E}}^{+}(\mathbf{r}, t)\right\rangle-2\left|\left\langle\hat{\boldsymbol{E}}^{-}(\mathbf{r}, t)\right\rangle\right|^{2},
$$

where $\hat{\boldsymbol{E}}^{-}=\left(\hat{\boldsymbol{E}}^{+}\right)^{\dagger}$ corresponds to the negative-frequency electric field. Eq. (1) links the field variance to the emitter correlation functions. We find the electric field fluctuations for one vector component (index suppressed for clarity)

$$
\begin{aligned}
\left\langle:\left[\Delta \hat{E}_{\theta}(\mathbf{r}, t)\right]^{2}:\right\rangle & =\left(\Delta \mathcal{E}_{1}\right)^{2}+\left(\Delta \mathcal{E}_{2}\right)^{2}+\left(\Delta \mathcal{E}_{12}\right)^{2} \\
\frac{\left(\Delta \mathcal{E}_{1}\right)^{2}}{2\left|g_{1}\right|^{2}} & =\rho_{33}+\rho_{44}-\left|\rho_{13}+\rho_{24}\right|^{2}-\operatorname{Re}\left[e^{2 \mathrm{i}\left(\theta+\phi_{1}\right)}\left(\rho_{31}+\rho_{42}\right)^{2}\right] \\
\frac{\left(\Delta \mathcal{E}_{2}\right)^{2}}{2\left|g_{2}\right|^{2}} & =\rho_{22}+\rho_{44}-\left|\rho_{12}+\rho_{34}\right|^{2}-\operatorname{Re}\left[e^{2 \mathrm{i}\left(\theta+\phi_{2}\right)}\left(\rho_{12}+\rho_{34}\right)^{2}\right] \\
\frac{\left(\Delta \mathcal{E}_{12}\right)^{2}}{2\left|g_{1} g_{2}\right|} & =2 \operatorname{Re}\left[e^{\mathrm{i}\left(\phi_{2}-\phi_{1}\right)} \rho_{23}+e^{-\mathrm{i}\left(2 \theta+\phi_{1}+\phi_{2, i}\right)} \rho_{14}\right]-4 \operatorname{Re}\left[e^{-\mathrm{i}\left(\theta+\phi_{1}\right)}\left(\rho_{13}+\rho_{24}\right)\right] \operatorname{Re}\left[e^{-\mathrm{i}\left(\theta+\phi_{2}\right)}\left(\rho_{12}+\rho_{34}\right)\right]
\end{aligned}
$$

In the present scheme, two-photon squeezing can be observed best in a balanced detection configuration, where $\left|g_{1}\right|^{2} /\left|g_{2}\right|^{2} \approx 1$. In very unbalanced situations, the signal is dominated by the single emitter terms [Eqs. (16) or (17)] and reduce the visibility of the cooperative squeezing term [Eq. (18)]. Nevertheless, we have confirmed numerically that the cooperative term can be discerned, as in the case of the incoherent fluorescence and the concurrence, for differences between the emitter's coupling constants up to an order of magnitude.

At weak driving near the two-photon resonance, we can derive a simple semianalytical formula following closely the approximations provided in the previous subsection for the concurrence. We assume again a cross-shaped density matrix, for which Eq. (15) reduces to

$$
\frac{1}{2}\left\langle:\left[\Delta \hat{E}_{\theta}(\mathbf{r}, t)\right]^{2}:\right\rangle \approx\left|g_{1}\right|^{2}\left(\rho_{33}+\rho_{44}\right)+\left|g_{2}\right|^{2}\left(\rho_{22}+\rho_{44}\right)+2\left|g_{1} g_{2}\right| \operatorname{Re}\left[e^{\mathrm{i}\left(\phi_{2}-\phi_{1}\right)} \rho_{23}+e^{-\mathrm{i}\left(2 \theta+\phi_{1}+\phi_{2}\right)} \rho_{14}\right] .
$$

Since the single-photon coherences are small near the two-photon resonance at weak drivings $\left(\left|\rho_{23}\right| \ll\left|\rho_{14}\right|\right)$, we can further simplify the squeezing signal. Thus for a balanced situation $\left|g_{1}\right|=\left|g_{2}\right|=|g|, \phi_{1}=\phi_{2}$ we obtain

$$
\frac{\left\langle:\left[\Delta \hat{E}_{\theta}(\mathbf{r}, t)\right]^{2}:\right\rangle}{2|g|^{2}} \approx \rho_{44}+\left(1-\rho_{11}\right)-2 \operatorname{Re}\left[e^{-2 \mathrm{i} \theta^{\prime}} \rho_{14}\right] \geq-2\left|\rho_{14}\right|,
$$

where we have redefined the quadrature angle $\theta^{\prime}=\theta+\left(\phi_{1}+\phi_{2}\right) / 2$ without loss of generality. Reduced electric field fluctuations are thus traced back to the two-photon coherence $\rho_{41}$ and are suppressed by any depopulation of the ground state and optimized for a quadrature angle such that $-2 \operatorname{Re}\left[e^{-2 \mathrm{i} \theta^{\prime}} \rho_{14}\right]=-2\left|\rho_{14}\right|$.

It is instructive to point out the connection to spin-squeezing, considering the fluctuations of the collective operators $S_{x}=\sum_{n}\left(\sigma_{n}^{\dagger}+\sigma_{n}\right) / 2, S_{y}=\sum_{n}\left(\sigma_{n}^{\dagger}-\sigma_{n}\right) / 2 \mathrm{i}$, and $S_{z}=\sum_{n} \sigma_{z, n}$. For driving near the two-photon resonance, we find that $\left\langle S_{x}\right\rangle \approx\left\langle S_{y}\right\rangle \approx 0$. Spin squeezing [15, 16] occurs when

$$
\xi_{x}=\frac{2\left(\left\langle S_{x}^{2}\right\rangle-\left\langle S_{x}\right\rangle^{2}\right)}{|\langle\boldsymbol{S}\rangle|}<1 .
$$

Direct algebraic manipulation shows that this is actually equivalent to the condition $\left\langle:[\Delta \hat{S}]^{2}:\right\rangle=\left(\left\langle: S_{x}^{2}:\right\rangle-\left\langle S_{x}\right\rangle^{2}\right)\langle 0$. Here,

$$
4\left\langle:[\Delta \hat{S}]^{2}:\right\rangle=1-\rho_{11}+\rho_{44}-2 \operatorname{Re}\left(\rho_{14}\right)+\left[\rho_{22}+\rho_{33}-2 \operatorname{Re}\left(\rho_{23}\right)\right] .
$$

Since the antisymmetric state is not driven, the last paranthesis vanishes recovering an expression that is proportional to Eq. 20 for vanishing quadrature phase and equal values of $g_{1}=g_{2}$.

[1] L. Knöll, S. Scheel, and D.-G. Welsch, "Coherence and statistics of photons and atoms," (Wiley, New York, 2001) Chap. QED in dispersing and absorbing dielectric media, pp. 1-60. 
[2] D. Martín-F, H. R. Haakh, K. Murr, and M. Agio, Phys. Rev. Lett. 113, 263605 (2014)

[3] H. T. Dung, L. Knöll, and D.-G. Welsch, Phys. Rev. A 64, 013804 (2001).

[4] L.-W. Li, P.-S. Kooi, M.-S. Leong, and T.-S. Yee, IEEE Trans. Microw. Theory Techn. 42, 2302 (1994).

[5] D. R. Lide, ed., CRC Handbook of Chemistry and Physics, 87th ed. (CRC Press, Boca Raton, FL, 2006).

[6] Z. Ficek and R. Tanas, Phys. Rep. 372, 369 (2002)

[7] H. T. Dung, L. Knöll, and D.-G. Welsch, Phys. Rev. A 66, 063810 (2002).

[8] H. J. Carmichael, Statistical Methods in Quantum Optics, 2nd ed. (Springer-Verlag, Berlin, Heidelberg, 2002).

[9] A. González-Tudela, D. Martin-Cano, E. Moreno, L. Martin-Moreno, C. Tejedor, and F. J. Garcia-Vidal, Phys. Rev. Lett. 106, 020501 (2011)

[10] C. Hettich, C. Schmitt, J. Zitzmann, S. Kühn, I. Gerhardt, and V. Sandoghdar, Science 298, 385 (2002).

[11] G. V. Varada and G. S. Agarwal, Phys. Rev. A 45, 6721 (1992)

[12] W. K. Wootters, Phys. Rev. Lett. 80, 2245 (1998)

[13] T. Yu and J. Eberly, Quant. Inf. Comp. 7, 459 (2007), ArXiv:quant-ph/0503089.

[14] P. D. Drummond and Z. Ficek, eds., Quantum Squeezing (Springer-Verlag, Berlin, Heidelberg, 2010).

[15] H. Saito and M. Ueda, Phys. Rev. Lett. 79, 3869 (1997).

[16] K. Hammerer, A. S. Sørensen, and E. S. Polzik, Rev. Mod. Phys. 82, 1041 (2010). 